\title{
LA INVESTIGACIÓN CUALITATIVA Y EL DESARROLLO DEL CONOCIMIENTO EN ENFERMERÍA ${ }^{1}$
}

\author{
Carmen de la Cuesta Benjumea
}

\footnotetext{
${ }^{1}$ Este artículo está basado en la Lección Inaugural del Doctorado en Ciencias de Enfermería, Departamento de Enfermería, Universidad Federal de de Santa Catarina, Florianópolis, en Agosto 2010.

2 PhD. Profesora Visitante del Departamiento de Salud de la Escuela Universitaria de Enfermería. Universidad de Alicante. Alicante, Espanha. E-mail: ccuesta@ua.es
}

\begin{abstract}
RESUMEN: La investigación es el crucial para el desarrollo disciplinar de enfermería y la investigación cualitativa genera un tipo de conocimiento que permite generalizaciones relacionadas con el fenómeno en estudio. El artículo primero se centra en la disciplina de enfermería entendiéndola como una ciencia humana, orientada hacia la práctica cuyo fenómeno central es el cuidado. El conocimiento de la disciplina, surge de la relación recíproca entre la teoría, el trabajo empírico y la práctica. Esta relación, permite a los profesionales enfrentar de mejor manera sus obligaciones con la sociedad. Respecto a la investigación cualitativa, el artículo plantea que el conocimiento que proviene de estos estudios permiten intervenciones de enfermería eficaces, basadas realmente en las necesidades del paciente y contextualizadas en situaciones específicas. Con un ejemplo, se ilustra como la investigación cualitativa produce un conocimiento científico que fortalece la práctica de enfermería y por ende, contribuye al desarrollo disciplinar.
\end{abstract}

DESCRIPTORES: Investigación en enfermería. Investigación cualitativa. Teoría de enfermería.

\section{QUALITATIVE RESEARCH AND DEVELOPMENT OF NURSING KNOWLEDGE}

\begin{abstract}
Research is crucial for the development of the nursing discipline and qualitative research produces a kind of knowledge that permits generalizations relating to the phenomenon under study. The paper first focus on the discipline of nursing considered as a human science, oriented towards practice which its central phenomena is care. The disciplinary knowledge arises out of a reciprocal relationship between theory, empirical work and practice. This relationship permits professional to meet in better manner their obligations to society. Relating to qualitative research, the paper argues that the knowledge coming from these studies permit effective nursing interventions truly based in patient's needs and contextualized in specific situations. With an example is illustrated how qualitative research produces scientific knowledge that strengthens nursing practice and therefore, contributes to disciplinary development.
\end{abstract}

DESCRIPTORS: Nursing research. Nursing theory. Qualitative research.

\section{PESQUISA QUALITATIVA E DESENVOLVIMENTO DO CONHECIMENTO EM ENFERMAGEM}

RESUMO: A pesquisa é o aspecto crucial para o desenvolvimento da disciplina de enfermagem e a pesquisa qualitativa gera um tipo de conhecimento que permite generalizações relacionadas com o fenômeno em estudo. $\mathrm{O}$ artigo focaliza inicialmente a disciplina de enfermagem entendendo-a como uma ciência humana, orientada para a prática cujo fenômeno central é o cuidado. O conhecimento da disciplina surge da relação recíproca entre a teoria, o trabalho teórico e a prática. Esta relação permite que os profissionais enfrentem melhor suas obrigações para com a sociedade. Com relação à pesquisa qualitativa, o artigo argumenta que o conhecimento que provém desses estudos permite intervenções de enfermagem eficazes, baseadas realmente nas necessidades do paciente e contextualizadas em situações específicas. Um exemplo ilustra como a pesquisa qualitativa produz um conhecimento científico que fortalece a prática de enfermagem e, portanto, contribui para o desenvolvimento da disciplina

DESCRITORES: Pesquisa em enfermagem. Teoria de enfermagem. Pesquisa qualitativa 


\section{INTRODUÇÃO}

En este artículo argumento que la investigación es el motor del desarrollo disciplinar y en concreto me centro en la investigación cualitativa, aquella que se interesa por lo específico, por las particularidades y no las generalidades; la que revela fenómenos muy cercanos a la práctica y disciplina de enfermería. Así el conocimiento que revela la investigación cualitativa es ideográfico; esto es, aquel que permite generalizaciones relacionadas con el caso o fenómeno en estudio.

\section{Ciencia y disciplina}

Antes abordar el tema de la investigación cualitativa y su relevancia para el desarrollo de conocimiento en enfermería, debemos preguntarnos si ésta es una ciencia, y si es así, ¿de qué clase?

Una gran teórica de enfermería ${ }^{1}$ explica que la enfermería es una ciencia humana, en tanto que se ocupa de la experiencia de los seres humanos y de asuntos concernientes a la salud-enfermedad. Aunque se use conocimiento básico y aplicado para lograr sus objetivos, es una disciplina orientada hacia la práctica, sus miembros buscan el conocimiento sobre las respuestas humanas a las situaciones de salud-enfermedad. El objetivo del desarrollo del conocimiento, es comprender las necesidades de cuidados de las personas, y aprender a como mejor cuidar de ellas. Es también, una disciplina orientada hacia la salud, sin desconocer la atención directa a los enfermos, la salud se entiende como una parte integral de la enfermería.

Lo que la hace distinta de otras disciplinas de la salud, es que es una disciplina del cuidado, constituyendo este el fenómeno central disciplinar. ${ }^{1}$ Nociones sobre la enfermedad, procesos mórbidos, síntomas, lesiones, técnicas o procedimientos, se sitúan en la periferia, y abren paso a un concepto integrador del quehacer en enfermería que está centrado en la persona, esto es: los cuidados.

Sobre los cuidados se ha escrito mucho, y por medio del desarrollo de conceptos y la investigación, se está enriqueciendo su significado, revelando sus elementos. Cuidar es todo lo que ayuda a vivir y permite existir. ${ }^{2}$ Su objetivo va mas allá de la enfermedad, se dirigen a todo lo que estimula la vida. Hoy en día se entiende como una relación y como un proceso; y no consiste en la mera ejecución de tareas o la realización de procedimientos aunque no las excluye. Así, la disyuntiva entre cuidado y técnica es aparente y artificial. Por ejemplo, se ha planteado, que el confort del paciente comprende tanto los cuidados como la habilidad técnica para dispensarlos. Este confort tiene dos componentes: uno, el que motiva a iniciar el proceso de enfermería y proporciona la calidad humanística durante los procedimientos; y el otro que consiste en los propios procedimientos de enfermería. ${ }^{3}$

El cuidado de enfermería es continuo y no esporádico o puntual. Es un proceso de interacción, con aspectos invisibles, donde la cercanía a la experiencia subjetiva del paciente es necesaria y proporciona una base para una relación eficaz entre el profesional de enfermería y el paciente. ${ }^{4}$ Por ello, requiere del conocimiento subjetivo, esto es, del conocimiento que se revela por los métodos de investigación cualitativos.

Por su lado, la disciplina de Enfermería, comprende las teorías que describen, explican y prescriben sobre el fenómeno central de su interés, el cuidado, así como los resultados de las investigaciones que se relacionan con este fenómeno. ${ }^{1}$ Además, integra aquellas otras disciplinas tales como la Antroplogía, la Biología y la Psicología, que son esenciales para su continuo desarrollo y para el desempeño de sus miembros. ${ }^{1}$ Por tanto, la enfermería es hoy en día, una disciplina plural, diversa y en crecimiento. Hemos superado la falsa dicotomía si las teorías son de enfermería o para la enfermería, es decir si la enfermería es una ciencia básica o aplicada; por tanto, ya no se discute si el desarrollo teórico debe concentrarse en lo que hace a la disciplina única o si por el contrario debe estar enfocado hacia la practica clínica. El objetivo de la teoría enfermera es hoy, contribuir al acervo de conocimiento que se requiere para la práctica clínica en una gran variedad de situaciones. Creo que es en la práctica donde se dirimen las cuestiones y donde queda patente lo que la hace única frente a las demás disciplinas de la salud y lo que comparte con ellas.

Hasta llegar aquí, ha pasado tiempo donde las enfermeras han ido conquistando espacios en el mundo académico y científico. Primero, fueron la teóricas de la enfermería, quienes elaboraron sus modelos y teorías de manera positivista, acorde a como en su época, se construía el saber científico. Luego, llegó el proceso de atención de enfermería, que propuso una revolución de la práctica y trajo aparejado los diagnósticos de enfermería. Por ultimo, el escenario actual, hecho de múltiples teorías, perspectivas, métodos, donde el conocimiento cualitativo está desarrollando teorías de rango medio, cercanas a la realidad, esto es: específica y situadas. Estas teorías nos muestran que en enfermería, la 
teoría es una base para la autonomía profesional y fuente para el conocimiento clínico.

Pero si bien es cierto, hasta bien entrados los años 80, la propuestas teóricas de enfermería, ya fueran modelos, teorías, marcos conceptuales, técnicas para desarrollar conceptos y diagnósticos enfermeros, estaban basadas, orientadas e instrumentalizadas por el positivismo y los métodos cuantitativos. Otra teórica ${ }^{5}$ en 1984 rompe significativamente esta tendencia con su estudio fenomenológico sobre el ejercicio experto de enfermería y posteriormente, con su teoría sobre los cuidados. Aquí, se inaugura una nueva época, plural, en la que tuvieron lugar y se continúan dando, desarrollos muy significativos tales como, una obra sobre las transiciones, ${ }^{6}$ y el desarrollo de conceptos en enfermería a través de métodos cualitativos.

Así, solapándose con este giro hacia lo cualitativo, irrumpe en el escenario teórico el análisis de conceptos. Los conceptos son los elementos de la teoría, de ahí su importancia en el desarrollo disciplinar. Implica la síntesis de puntos de vista sobre un concepto y su distinción de otros. ${ }^{7}$ Junto a la meta síntesis, y el meta análisis, conforman las estrategias con las que contamos hoy en día para la síntesis del conocimiento. Entre los conceptos de interés para la enfermería analizados, cabe mencionar los de empatía, aislamiento social, luto, integración, alianzas terapéuticas, aflicción crónica, y políticas de salud. Para ilustra esto comentaré brevemente en el análisis del concepto central a la disciplina de enfermería. Se encontró que en muchas teorías de enfermería, hay diferentes perspectivas implícitas sobre el cuidado, y que por tanto, se necesitaba una clarificación de este concepto. ${ }^{8}$ Lo analizaron de forma pragmática, revisando críticamente la bibliografía; encontraron cinco maneras de conceptualizarlo, y que algunos autores relacionaban más de una manera en sus modelos. Así, el cuidado en la bibliografía se conceptualiza: Como un rasgo humano; Como un imperativo moral; Como afecto que se manifiesta a través de sentimientos emocionales o empatía y sentimientos de compromiso; Como la relación entre la enfermera, y el paciente; Como una intervención terapéutica.

Además, las autoras hallaron dos resultados: uno que se expresa en la experiencia subjetiva del paciente y el otro que se manifiesta en su respuesta física. Este trabajo ya indica, que el concepto de cuidado, como el de salud, es polisémico. ${ }^{8}$

Estas investigadoras, continuaron profundizando en el concepto y hallaron que el cuidado entre individuos estaba claramente descrito, pero entre familias o comunidades estaba poco explicado. Su análisis clarificó la situación, reveló vacíos que no se habían reconocido, insuficiencias y áreas en las que se necesitaba mas trabajo cualitativo. No se llegó a una síntesis, pero se reveló información sobre la idoneidad del concepto para la investigación y su uso en la practica. ${ }^{8}$

Dado que la enfermería es una disciplina que se práctica y la investigación la entiendo como el motor del desarrollo disciplinar, seguidamente abordo la investigación en su relación con la práctica de enfermería.

\section{La investigación}

Tanto en el desarrollo conceptual, como en e campo clínico, lo que ha venido dominado en la investigación enfermera ha sido la búsqueda de explicaciones causales, que se puedan generalizar a grupos de pacientes o situaciones de cuidados. Pero desde los años 90, han irrumpido con fuerza, en el área de la salud, métodos de investigación denominados cualitativos, que buscan la comprensión de fenómenos complejos y cambiantes, como son los relativos a las cuestiones de salud. Aquí, es importante anotar que la enfermería se ha interesado, desde el inicio, por esta nueva manera de representar el mundo.

Si analizamos las características de su práctica, podemos comprender porqué ha tenido una aceptación tan grande entre nosotras. La definición de una teórica ${ }^{9}$, aceptada como la definición del Consejo Internacional de Enfermeras (CIE), nos da una de las claves más importantes: la enfermería se coloca en la subjetividad de las personas para identificar sus necesidades. En la práctica, se trabaja con un método inductivo y enfocado en las personas. Debido al contacto permanente con el paciente, el profesional de enfermería está cerca de la experiencia humana y no de la enfermedad como un fenómeno etio-patogénico. La investigación pone su interés y compromiso por revelar el punto de vista del otro, del que es desconocido y no ha tenido voz autorizada. No es ajena a la experiencia cotidiana de la enfermería y quizás, por ello, se acerquen de manera intuitiva a este modo de investigar tantos profesionales.

Con frecuencia he escuchado a enfermeras, decir que para su práctica necesitan de otro conocimiento, que sienten que hay algo más por captar en las situaciones de cuidado y que muchas veces sus intervenciones se quedan cortas. En efecto, 
la práctica influye en el desarrollo disciplinar al indicar áreas y maneras de indagar. Así, el conocimiento de la disciplina, proviene de una relación recíproca entre la teoría, el trabajo empírico y la práctica. ${ }^{10}$ Esta relación, permite a los profesionales enfrentar de mejor manera sus obligaciones con la sociedad. ${ }^{11}$ Ilustremos esto con un ejemplo.

Una enfermera Neo Zelandesa ${ }^{12}$ relata, que durante los 12 años que trabajó en la Unidad de Cuidados Intensivos, se dieron numerosas ocasiones en las que le fue imposible preguntar a los pacientes cómo se sentían, o qué es lo que necesitaban para ayudarles a disminuir su sufrimiento, o a hacer frente a sus situaciones. Diariamente, experimentó la dificultad y frustración al intentar comunicarse de manera eficaz con ellos y de proporcionar cuidados sin estar completamente segura de sus necesidades y deseos. Fue esta falta de saber y la necesidad de comprender mejor las experiencias de los pacientes, lo que motivó su estudio en que el que se preguntó: ¿Cuál es la vivencia de los pacientes, en la Unidad de Cuidados Intensivos?

La misma autora ${ }^{12}$ encontró que estar $\sin v o z$, es una de las características más significativas de la experiencia de estar críticamente enfermo en la Unidad de Cuidados Intensivos (UCI). En su estudio detalla la naturaleza de esta experiencia. Así, por ejemplo, narra que sin la habilidad de hablar, los pacientes se sienten como si estuvieran solos en un mundo a parte del mundo de los otros. Esto se ilustra cuando comenta que para los pacientes, es importante escuchar las voces de los otros, particularmente de los familiares pero que es más significativo poder responderles. La experiencia de estar en la UCI se hace soportable con la presencia de los otros, amigos y familiares. Esta presencia, confirma su existencia, y les permite sentir que alguien está ahí, con ellos y por ellos. En el estudio se señala, que mientras estar acompañado es mejor que estar solo, estar con quienes les conocen y aman, proporciona a los pacientes un sentimiento de seguridad y continuidad. Se revela que, en ausencia de la familia, las enfermeras, con frecuencia, son las únicas personas que proporcionan a estos pacientes una información valiosa sobre su situación. Por último, el estudio también encuentra que los pacientes en la UCI, en algún momento de su experiencia, sienten que se van a morir por lo insoportable y permanente de sus síntomas; la muerte se convierte para ellos, en una presencia contundente.

En las conclusiones de su trabajo, el autor ${ }^{12}$ señala que es esencial que las enfermeras, y los que cuidan de pacientes críticos, comprendan la naturaleza de su experiencia. Sin esta apreciación profunda y subjetiva, se puede subestimar la magnitud de los trastornos y el peso abrumador que tienen para los pacientes las privaciones físicas y emocionales. La investigadora enfatiza, que se ha ser sensible y estar muy atento a sus necesidades, y a las de sus familiares; en su opinión, esto significa trascender el ambiente tecnológico de la UCI para observar y responder a la vulnerabilidad y fragilidad humana. Sugiere, que las intervenciones de enfermería, deben reconocer y fortalecer las capacidades individuales, así como, los sistemas de apoyo sociales.

Bien, a simple vista, este estudio no ha hecho grande aportes teóricos a la disciplina, pero ha visibilizado la situación de unos pacientes. Con ello, ha abierto la puerta a intervenciones de enfermería eficaces, basadas realmente en las necesidades del paciente y contextualizadas en situaciones específicas. Al hilo de esto, me viene a la memoria lo que dice una estudiosa ${ }^{13}$ sobre la importancia de que la enfermería cuente con este conocimiento en su práctica. Afirma que una enfermera con corazón, esto es sensible al otro, puede que no influya sobre el efecto de un antibiótico, pero para el paciente, esta será, sin duda, una experiencia cualitativamente diferente. ${ }^{13}$ Los que hemos sido pacientes compartimos plenamente sus palabras.

La ciencia de enfermería es humana, todo lo que revele la humanidad presente en las situaciones de cuidados, nos hará sin duda, mejores profesionales. La investigación cualitativa, así, produce un conocimiento científico que fortalece la práctica de enfermería y por ende, contribuye al desarrollo disciplinar.

\section{CONCLUSIÓN}

Concluyendo, el objetivo del desarrollo del conocimiento en enfermería es comprender las necesidades de las personas y aprender a como mejor cuidar de ellas. La necesidad de teorías de rango medio, sustantivas, cercanas a la realidad práctica y cotidiana, ya se ha expresado en diversos foros académicos. Los grandes modelos, con poca base empírica son cosa del pasado. Hoy la enfermería, necesita un conocimiento que le permita abordar, de manera eficiente y moral la complejidad y variedad de situaciones a las que se enfrenta; un conocimiento inductivo, cercano y situado. Este conocimiento, sin duda cerrará brechas, hará que la práctica y la disciplina estén, en verdad, interrelacionadas. 
Hemos recorrido un largo camino, la historia de la Enfermería es rica. La formación es solo una etapa, no debe significar un punto de llegada sino de partida, como dice el poema Itaca de Cavafis, para otros puertos, donde nos aguardarán nuevas experiencias,. Si bien la investigación es esencial para el desarrollo del conocimiento enfermero, la educación de los que la practican con una perspectiva enfermera es de vital importancia, sino cómo plantear preguntas de investigación relevantes para la práctica de la enfermería? Cómo hacer que el conocimiento disciplinar crezca sin una mirada enfermera?

Bien, el futuro disciplinar está en nuestra manos. El desafío es ir desarrollando la disciplina al mismo tiempo que ensanchamos sus fronteras, el campo disciplinar no está ni mucho menos cerrado recién lo estamos construyendo pero esta construcción no es excluyente ni alejada del sentir de las comunidades, debe estar en sintonía con las poblaciones objeto de nuestros cuidados y en dialogo con las disciplinas que intervienen en el campo de la salud. La apuesta, como siempre es a la diversidad y a la apertura.

En la formación se asienta el desarrollo disciplinar, por ello es fundamental que llevemos a cabo lo aprendido y nos comprometamos, desde diferentes niveles, con la investigación que facilite una practica de enfermería sensible a las realidades de las personas a las que cuidamos, a su diversidad cultural y a los contextos en los que se desenvuelven sus vidas. A los investigadores noveles, les insto a que planteen imaginativas y provocativas preguntas de investigación, que empujen las fronteras del conocimiento enfermero.

\section{REFERENCIAS}

1. Meleis A. Theoretical nursing. development and progress. $4^{\text {a }}$ ed. Philadelphia (US): Lippincott; 2007.
2. Colliére MF. Encontrar el sentido original de los cuidados enfermeros. Rev Rol de Enfermería. 1999 Jan; 22(1):27-31

3. Field PA, Morse JM. Nursing research: the application of qualitative approaches. London (UK): Croom Helm, 1985.

4. Zichi-Cohen M. The experience of surgery: phenomenological clinical nursing research. In: Omery A, Kasper, CE, Gayke G, Editors. In search of nursing science. Thousand Oaks (US): SAGE, 1995. p. 159-74.

5. Benner P. From novice to expert. California (US): Addison Wesley, 1984.

6. Meleis AI, Sawyer LM, Im EO, Schumacher K, Meleis A. Experiencing transitions: an emerging middle-range theory. ANS Adv Nurs Sci. 2000 Sep; 23(1):12-28.

7. Knafl KA, Deatrick JA. Knowledge synthesis and concept development in nursing. In: Rodgers BL, Knafl KA, Editors. Concept development on nursing. Philadelphia (US): Saunders; 2000. p. 39-54.

8. Morse JM. Exploring pragmatic utility: concept analysis by critically appraising the çiterature. In: Rodgers BL, Knafl KA, Editors. Concept development on nursing. Philadelphia (US): Saunders; 2000. p.333-52.

9. Henderson V. Basic principles of nursing care. Geneva: International Council of Nurses; 1979.

10. Dean H. Science and practice. In: Omery A, Kasper CE, Gayke G. Search of nursing science. Thousand Oaks (US): SAGE; 1995. p.275-90

11. Donalson SK. Introduction: nursing science for nursing practice In: Omery A, Kasper CE, Gayke G. Search of nursing science. Thousand Oaks (US): SAGE; 1995.p.3-12.

12. Parker V. On confronting life and death. In: Madjar I, Walton JA. Nursing and the experience of illness. NSW (AU): Allen \& Unwin; 1999.p. 57-76

13. Madjar, I.Inflingir y Manejar el dolor: un desafío para la Enfermería. Invest Educ Enferm. 2000; 1 (1):37-47 